
МЕТОДИКИ ДИАГНОСТИКИ КОПИНГ-ПОВЕДЕНИЯ

\author{
Луговская A. A. (lug.al@mail.ru)
}

УО «Гродненский государственный медицинский университет», Гродно, Беларусь

Введение. Интерес к проблеме измерения совладающего поведения, или копинг-поведения, обусловлен требованиями современной психометрики. На данный момент в клинике отсутствуют методики диагностики данного конструкта, обладающие обоснованными психометрическими характеристиками.

Цель. Провести анализ психометрических свойств икаль конструктивных стратегий опросника копинг-поведения на основе модели Раша.

Материал и методы. С помощью методики диагностики копинг-поведения обследованы 270 человек. По результатам исследования были рассчитаны меры трудности утверждений шкаль конструктивных стратегий опросника, оценена конструктная валидность утверждений икаль методики, определены показателей надежности и сепарационной статистики.

Результатыл. Наблюдаемье значения мер трудности утверждений шкаль опросника распределились 6 диапазоне от -1,04 до 1,20 логита. Значения индексов UMS и WMS пунктов имели приемлемые значения (в диапазоне 0,7-1,3). Индекс надежности составил 0,81.

Bыводы. Определено, что утверждения внутри шкаль моделируемого опросника сбалансированы по степени трудности. Конструктная валидность пунктов на достаточном уровне. Показатель надежности методики имеет приемлемое значение $(0,81)$. Применение опросника позволяет диагностировать три уровня выраженности исследуемого конструкта.

Ключевые слова: методика диагностики копинг-механизмов, конструктная валидность, надежность, модель Раша

\section{Введение}

Разработка психодиагностических методик, отвечающих требованиям современной психометрики, является актуальной и требующей особого внимания проблемой современной психодиагностики. Речь идет о значимости таких показателей, как валидность, надежность и внутренняя согласованность психодиагностического инструмента [1]. Возрастающая роль психосоциальной терапии и реабилитации в психиатрии и психотерапии обуславливает усиленный интерес к анализу совладающего, адаптивного поведения, или копинг-поведения у пациентов с психическими и поведенческими расстройствами [2].

На данный момент в клинике для диагностики данного конструкта в основном используются переводные шкалы и опросники, адаптированные на русскоязычных выборках и разработанные в рамках классической теории тестов. Однако последняя не удовлетворяет требованиям современного фундаментального научного измерения $[1,3,4]$.

Одной из теорий создания и стандартизации методик, которая соответствует требованиям современной психометрики, является модель Раша. Данная модель определяет технологию тестов, опираясь на вычисление меры трудности утверждения методики и уровня выраженности измеряемого конструкта. Модель Раша утверждает, что вероятность дать ключевой ответ на утверждение теста зависит только от уровня выраженности исследуемого свойства у испытуемого и от уровня трудности тестового утверждения (т.е. количественной характеристики утверждения теста, не зависящей от выборки испытуемых). Данный статистический метод обеспечивает также трансформацию полученных в процессе тестирования результатов в интервальную шкалу - шкалу логитов $[5,6]$.

Цель данного исследования - анализ психометрических свойств шкалы конструктивных стратегий опросника копинг-поведения на основе модели Раша.

\section{Материал и методы}

В исследовании принимали участие пациенты с верифицированными диагнозами из рубрик невротические, связанные со стрессом и соматоформные расстройства (F40, F41, F43, F45), аффективные расстройства (F32, F33) отделения пограничных состояний УЗ ГОКЦ «Психиатрия-Наркология» $(\mathrm{n}=150)$ и здоровые испытуемые из общей популяции $(\mathrm{n}=150)$. Пациенты, страдающие перечисленными расстройствами, и здоровые интервьюируемые в рамках настоящего исследования рассматриваются как однородная группа, так как для моделирования по методике Раша требуется широкий диапазон распределения мер выраженности исследуемого конструкта (от минимума до максимума). Coстав группы по полу: мужчины -119 , женщины - 151. Возраст испытуемых в диапазоне от 18 до 55 лет.

В качестве стимульного материала для диагностики копинг-поведения использовались утверждения методики диагностики копинг-механизмов Э. Хайма. Испытуемым предлагались 26 утверждений данного опросника с инструкцией по оценке своего поведения в стрессовых ситуациях и ситуациях высокого эмоционального напряжения [2, 7]. Нами была использована ранговая система ответов. Порядок ранжирования категорий ответа соответствует возрастанию частоты использования копинг-стратегии. 
Соответственно, каждая категория ответа на каждый диагностический пункт приобретает собственную меру трудности. Чем больше значение трудности пункта, тем меньше вероятность предоставления ответа с более высокой оценкой $[5,8]$.

На этапе идентификации конструктов нами проведен факторный анализ. Использовался метод главных компонент с процедурой ортогонального вращения Varimax. Анализ факторной структуры моделируемого опросника выявил, что утверждения не факторизуются в оригинальную трехфакторную версию: поведенческую (пункты № 1.1-1.8), когнитивную (пункты № 2.1-2.10) и эмоциональную (пункты № 3.1-3.8). Для решения вопроса о количестве факторов была проведена оценка собственных значений выделенных факторов и принято решение о двухфакторной структуре методики диагностики копинг-поведения. На основе анализа значений факторных нагрузок на выделенные факторы были сформированы две шкалы.

В данной статье приводятся результаты анализа психометрических свойств шкалы конструктивных стратегий методики диагностики копинг-поведения.

Основным статистическим методом работы был политомический вариант модели Раша. Данная модель представляет собой систему математических методов построения измерительных шкал для оценки латентных конструктов.

В нашем исследовании для определения психометрических характеристик шкалы конструктивных копинг-стратегий были последовательно проведены основные этапы Раш-анализа.

На первом этапе на основе имеющейся матрицы данных, содержащей ответы всех испытуемых на все утверждения шкалы методики, были рассчитаны меры трудности пунктов и определены меры выраженности исследуемого конструкта. Выраженность конструкта определяется как доля ключевых ответов конкретного испытуемого от его ответов на все утверждения, а трудность утверждения - как доля неключевых ответов от ответов всех испытуемых на конкретный пункт. Далее данные значения трансформировались в шкалу логитов, что обеспечивало возможность представить эти параметры на общей шкале, среднее значение которой равно 0. Следовательно, респондент, получивший показатель выраженности признака в 0 логитов, обладает средней выраженностью признака, а утверждение, имеющее по критерию трудности 0 логитов, оценивается как имеющее среднюю трудность. Благодаря данным статистическим процедурам, в модели Раша предоставляется возможность оценки диагностической точности анализируемой измерительной шкалы $[3,6,8]$.

На этапе оценки качества шкалы конструктивных копинг-стратегий были рассчитаны индексы качества для каждого утверждения. К ним относятся невзвешенное среднее квадратичное - UMS, взвешенное среднее квадратичное WMS. Данные индексы позволяют оценить конструктную валидность каждого утверждения, то есть определяют, насколько эффективно пункт шкалы функционирует в оценке исследуемого конструкта (в случае настоящего исследования - копинг-стратегии). Анализ индексов качества утверждений позволяет делать выводы и о конструктной валидности всего диагностического инструмента. Для рейтинговых шкал диапазон приемлемых значений индексов UMS и WMS составляет 0.7-1.3 [4, 8].

Оценка надёжности шкалы конструктивных стратегий моделируемой методики диагностики проводилась через анализ показателей сепарационной статистики. В модели Раша это показатели надёжности и Индекс числа слоев. Показатель надежности концептуально близок коэффициенту альфа-Кронбаха и имеет такие же нормативные диапазоны. Индекс числа слоев представляет собой количество клинически значимых уровней выраженности исследуемого латентного свойства (копинга), которое способна выявить шкала как диагностический инструмент в исследуемой выборке. Приемлемыми считаются значения, превышающие 1, при этом большие значения характеризуют большую надёжность [9].

\section{Результаты и обсужндене}

В результате применения алгоритма политомического варианта модели Раша были рассчитаны параметры трудности утверждений, произведена оценка соответствия распределения трудностей ответных категорий утверждений распределению мер выраженности копинга, выполнен анализ показателей конструктной валидности и надёжности шкалы конструктивных копинг-стратегий.

В табл. 1 представлены утверждения методики, сформировавшие шкалу конструктивных копинг-стратегий.

Tаблица 1. - Утверждения шкалы конструктивных копинг-стратегий

\begin{tabular}{|l|l|}
\hline$№$ & Утверждения \\
\hline 1.1 & Я погружаюсь в любимое дело, стараясь о них забыть \\
\hline 1.2 & $\begin{array}{l}\text { Я стараюсь помочь людям и в заботах о них я забываю о своих } \\
\text { горестях }\end{array}$ \\
\hline 1.3 & $\begin{array}{l}\text { Стараюсь не думать, всячески избегаю думать о своих } \\
\text { неприятностях }\end{array}$ \\
\hline 1.5 & $\begin{array}{l}\text { Чтобы пережить эти трудности, я берусь за осуществление своей } \\
\text { давней мечты (например путешествия) }\end{array}$ \\
\hline 2.1 & $\begin{array}{l}\text { Говорю себе: в данный момент есть что-то более важное, чем эти } \\
\text { трудности }\end{array}$ \\
\hline 2.2 & Говорю себе: это судьба, нужно с этим мириться \\
\hline 2.3 & $\begin{array}{l}\text { Говорю себе: это не существенные, не так все плохо, в основном все } \\
\text { хорошо }\end{array}$ \\
\hline 2.5 & $\begin{array}{l}\text { Я стараюсь проанализировать, все взвесить и объяснить себе, что } \\
\text { же случилось }\end{array}$ \\
\hline 2.6 & $\begin{array}{l}\text { Я говорю себе: по сравнению с проблемами других людей мои - это } \\
\text { пустяк }\end{array}$ \\
\hline 2.9 & $\begin{array}{l}\text { Я придаю своим трудностям особый смысл, преодолевая их, я } \\
\text { совершенствую себя }\end{array}$ \\
\hline 2.10 & $\begin{array}{l}\text { В данное время я полностью не могу справиться с этими } \\
\text { трудностями, но со временем смогу справиться с ними и с более } \\
\text { сложными, чем ранее }\end{array}$ \\
\hline 3.4 & Я всегда уверен, что есть выход из трудной ситуации \\
\hline
\end{tabular}

В процессе анализа содержательной характеристики утверждений, сформировавших данную шкалу (табл. 1), было выявлено, что пункты шкалы имеют общий паттерн стратегий, направленный на диагностику конструктивного для личности копинг-поведения. 
На следующем этапе нашего исследования проведен статистический анализ исходной матрицы данных, содержащей баллы всех испытуемых по всем градациям утверждений шкалы конструктивных копинг-стратегий, и определены значения трудностей данных пунктов. При анализе значений показатели сопоставлялись с оптимальным для психодиагностических методик интервалом - от -2 до +2 логитов $[3,7,8]$.

По результатам оценки трудности всех утверждений было определено, что все пункты опросника находятся в пределах указанного диапазона. При этом утверждения имели разную степень трудности, от легких $(-1,04)$ до достаточно трудных $(1,20)$. Для «легких» пунктов шкалы (с невысокими значениями трудности) высока вероятность ключевых ответов у лиц с минимальной выраженностью использования активной копинг-стратегии. Например утверждение № 3.4 «Я всегда уверен, что есть выход из трудной ситуации» (трудность: -1,04). В то же время шкала содержит и «трудные» пункты, ключевой ответ для которых возможен только для тех испытуемых, у которых данная копинг-стратегия значительно выражена. Например утверждение № 1.5 «Чтобы пережить эти трудности, я берусь за осуществление своей давней мечты» (например путешествия) (трудность: 1,20). Основная масса утверждений шкалы конструктивных копинг-стратегий обладает средним уровнем трудности. Это характеризует пункты как обладающие способностью к диагностике среднего диапазона выраженности копинга. Но наличие в структуре шкалы конструктивных копинг-стратегий «полюсных» утверждений обеспечивает возможность оценки выраженности проявления исследуемого конструкта в достаточно широких границах.

Оценка соответствия распределения трудностей ответных категорий утверждений распределению мер выраженности копинга осуществлялась путем визуального анализа карты распределения мер трудности утверждений, сформировавших шкалу конструктивных копинг-стратегий, и мер конструкта. Так как в модели Раша меры трудности и меры конструкта оцениваются в одних и тех же единицах измерения (логитах), имеется возможность оценить данные на одной и той же интервальной шкале. Если распределение трудностей категорий пунктов полностью охватывает распределение мер выраженности исследуемого конструкта у испытуемых, шкала оценивается как обладающая достаточной диагностической мощностью $[3,8]$.

На рис. 1 представлена карта соотношения мер выраженности копинга и мер трудности утверждений, сформировавших шкалу конструктивных копинг- стратегий. Как следует из анализа данных карты, распределение мер выраженности копинга (столбиковая диаграмма) находится в диапазоне от $-3,8$ логитов до 2,5 логитов. Величина диапазона разброса мер выраженности копинга - 6,3 логита. Визуальный анализ распределения оценок испытуемых (левая часть карты) показывает незначительное смещение распределения в сторону умеренных и невысоких мер выраженности копинга.

Распределение категорий ответов на диагностические утверждения шкалы конструктивных копинг-стратегий отражено в правой части карты на рис.1. Как следует из оценки этой части, меры трудностей категорий ответов находятся в диапазоне от $-3,7$ логита до 3,9 логита. Величина диапазона составляет 7,6 логита, что на один «шаг» логита превышает величину диапазона мер выраженности копинга (6,3 логита). Из этого следует, что границы диапазона распределения мер трудности утверждений шире, чем границы диапазона распределения мер выраженности исследуемого конструкта.

Это позволяет сделать вывод, что распределение ответных категории пунктов шкалы конструктивных копинг-стратегий согласуется с распределением исследуемого конструкта в популяционной выборке. Основная масса категорий ответов на утверждения данной шкалы локализуются в диапазоне умеренной степени трудности (диапазон от -2 до 2 логитов). Большинство мер выраженности копинга у испытуемых располагается в диапазоне от $-1,3$ до 1,2 логита. Следует обратить внимание, что большинство категорий направлены на оценку умеренной выраженности копинга, а также на то, что в данной шкале присутствуют и «полюсные» утверждения.

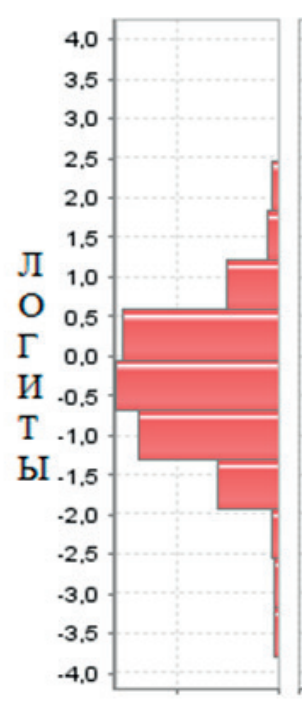

Распределение испытуемых

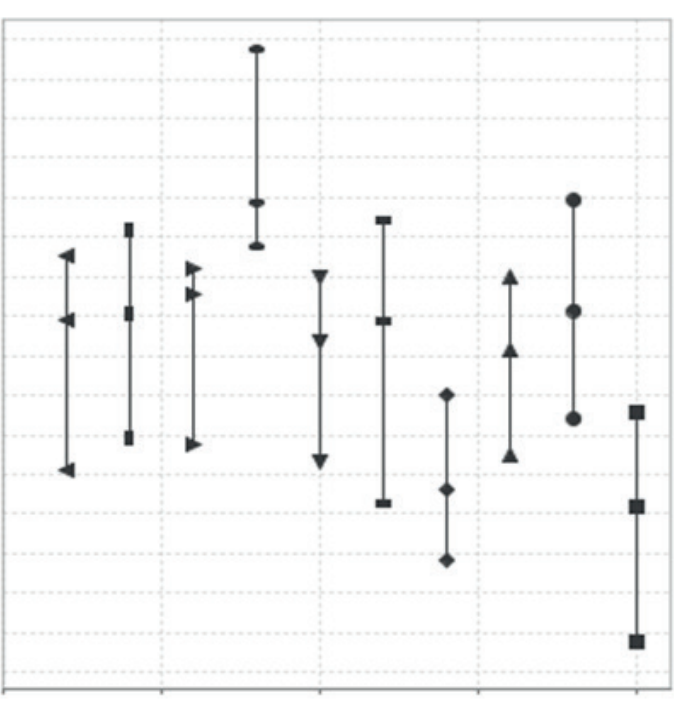

Распределение категорий пунктов
Рисунок 1. - Карта распределения мер выраженности конструкта у испытуемых и трудности категорий ответов на утверждения шкалы конструктивных стратегий опросника копинг-поведения 
В результате проведенного анализа данных (рис.1) можно сделать вывод, что диапазон распределения мер трудностей категорий утверждений полностью покрывает диапазон распределения мер выраженности копинга. Следовательно, шкалу можно охарактеризовать как способную к точной диагностике копинга в широком диапазоне выраженности исследуемого конструкта.

На этапе анализа конструктной валидности шкалы конструктивных копинг-стратегий были рассчитаны значения двух среднеквадратичных индексов валидности утверждений: UMS и WMS. Данные индексы качества отражают степень соответствия моделированных вероятностей ответов на пункты эмпирическим данным.

Анализ рассчитанных индексов качества после первого статистического цикла выявил утверждение, нарушающее конструктную валидность шкалы. Как видно из табл. 2, индекс UMS утверждения 2.10 («В данное время я полностью не могу справиться с этими трудностями, но со временем смогу справиться с ними и с более сложными, чем ранее») имеет значение, равное 1,34. Данный показатель превышает допустимый для диагностических методик диапазон. Это свидетельствует о том, что пункт с высокой степенью вероятности принадлежат к иному, нежели исследуемый, конструкту или характеризует общие проявления нескольких конструктов. Следовательно, он нарушает конструктную валидность всей шкалы и подлежит удалению. После элиминации данного пункта было проведено повторное моделирование, по результатам которого индекс UMS утверждения 2.2 («Говорю себе: это судьба, нужно с этим мириться») имел значение 1,37. Данная статистика характеризует пункт 2.2 как обладающий низкой конструктной валидностью. Как результат анализа, утверждение также было удалено из структуры шкалы. После проведения очередной итерации оставшиеся утверждения шкалы имели показатели индексов качества приемлемого диапазона. Итоговый вариант шкалы конструктивных копинг-стратегий был сформирован десятью утверждениями, имеющими удовлетворительные значения индексов валидности.

Tаблица 2. - Значения трудности и индексы качества (WMS, UMS) утверждений шкалы конструктивных стратегий

\begin{tabular}{|l|c|c|c|c|c|c|}
\hline \multirow{2}{*}{$\begin{array}{c}\text { № } \\
\text { вопроса }\end{array}$} & \multicolumn{3}{|c|}{ WMS } & \multicolumn{3}{c|}{ UMS } \\
\cline { 2 - 7 } & $\begin{array}{c}1-я \\
\text { итерация }\end{array}$ & $\begin{array}{c}2-я \\
\text { итерация }\end{array}$ & $\begin{array}{c}\text { 3-я } \\
\text { итерация }\end{array}$ & $\begin{array}{c}\text { 1-я } \\
\text { итерация }\end{array}$ & $\begin{array}{c}\text { 2-я } \\
\text { итерация }\end{array}$ & $\begin{array}{c}\text { 3-я } \\
\text { итерация }\end{array}$ \\
\hline 1.1 & 1,03 & 1,03 & 1,05 & 1,04 & 1,04 & 1,06 \\
\hline 1.2 & 0,91 & 0,92 & 0,98 & 0,92 & 0,94 & 0,99 \\
\hline 1.3 & 1,04 & 1,06 & 1,10 & 1,04 & 1,07 & 1,10 \\
\hline 1.5 & 1,02 & 1,01 & 1,03 & 0,98 & 0,95 & 0,96 \\
\hline 2.1 & 0,82 & 0,81 & 0,82 & 0,80 & 0,80 & 0,80 \\
\hline 2.2 & 1,13 & 1,16 & - & 1,17 & $\mathbf{1 , 3 7 *}$ & - \\
\hline 2.3 & 0,87 & 0,86 & 0,86 & 0,86 & 0,86 & 0,85 \\
\hline 2.5 & 0,98 & 1,01 & 1,00 & 0,97 & 1,00 & 0,98 \\
\hline 2.6 & 0,92 & 0,92 & 0,95 & 0,93 & 0,92 & 0,95 \\
\hline 2.9 & 1,02 & 1,08 & 1,11 & 1,07 & 1,20 & 1,23 \\
\hline 2.10 & 1,16 & - & - & $\mathbf{1 , 3 4}$ & - & - \\
\hline 3.4 & 1,03 & 1,06 & 1,04 & 1,03 & 1,06 & 1,04 \\
\hline
\end{tabular}

* - значения, не соответствуюшие диапазону приемлемьх
Надежность шкалы оценивалась с помощью показателей надежности и сепарационной статистики модели Раша $[3,8,9]$. Данные показатели рассчитывались отдельно для каждой итерации.

Показатель надежности по испытуемым после проведения первой итерации составил 0,71 , что соответствует хорошему уровню надежности шкалы методики (диапазон 0,7-0,9). Удаление из структуры шкалы утверждения 2.10 («В данное время я полностью не могу справиться с этими трудностями, но со временем смогу справиться с ними и с более сложными, чем ранее») улучшило характеристики показателя надежности $(0,75)$. Проведение третьего статистического цикла (после удаления утверждения 2.2 - «Говорю себе: это судьба, нужно с этим мириться») - позволило оценить надежность шкалы конструктивных стратегий на уровне 0,81. Каждая из проведенных итераций способствовала улучшению качества шкалы конструктивных копинг-стратегий по показателю надежности. Хороший уровень данного критерия по испытуемым свидетельствует о популяционной независимости вычисленных мер выраженности копинга, а методику характеризует как диагностически популяционно-независимую.

На следующем этапе нашего исследования был проведен анализ показателей Индекса слоев. После проведения первого статистического цикла меры выраженности копинга, воплощенные в суммарных баллах, разделились на 2 статистически значимых уровня выраженности исследуемого конструкта (Индекс числа слоев - 2,40). Удаление из структуры шкалы пунктов с низкой конструктной валидностью при повторных итерациях улучшило этот показатель сепарационной статистики (Индекс числа слоев - 2,65). Шкала, лишившись некачественных пунктов, значительно изменила свои дискриминативные возможности и способна дифференцировать 3 уровня выраженности исследуемого латентного свойства.

\section{Bblводы}

1. Утверждения внутри моделируемой шкалы конструктивных стратегий опросника копинг-поведения сбалансированы по степени трудности. Наблюдаемые значения трудностей пунктов достигают интервала 3 логитов (от -1,04 до 1,20 логита).

2. Шкала обладает достаточной диагностической мощностью. Величина диапазона распределения категорий ответов на диагностические утверждения составляет 7,6 логита.

3. Пункты шкалы методики диагностики копинг-поведения обладают достаточным уровнем конструктной валидности (значения индексов UMS и WMS всех утверждений в диапазоне 0.7-1.3).

4. Шкала конструктивных копинг-стратегий имеет хороший уровень надежности $(0,81)$.

5. Дифференциально-диагностические свойства шкалы обеспечивают возможность определения как минимум трех статистически значимых уровней выраженности копинга. 


\section{Литература}

1. Фер, Р. М. Психометрика. Введение / Р. М. Фер, В. Р. Бакарак ; пер. с англ. А. С. Науменко, А. Ю. Попова. - Челябинск : Изд. центр ЮУрГУ, 2010. -445 c.

2. Набиуллина, Р. Р. Механизмы психологической защиты и совладания со стрессом (определение, структура, функции, виды, психотерапевтическая коррекция) : учеб. пособие / Р. Р. Набиуллина, И. В. Тухтарова. Казань, 2003. - С. 17-25.

3. Ассанович, М. А. Оптимизация шкалы оценки депрессии Гамильтона на основе модели Раша [Электронный peсурс] // Медицинская психология в России. - 2015. № 2 (31). - C. 7. - Режим доступа: http://www.medpsy. ru/mprj/archiv global/2015 2 31/nomer09.php. - Дата доступа: 20.10.2016.

4. Ассанович, М. А. Статистическое обоснование критериев оценки выраженности измеряемого конструкта в клинической психодиагностике (на примере теста нервно-психической адаптации) / М. А. Ассанович // Психиатрия, психотерапия и клиническая психология. - 2014. - № 2 (16). - C. 9-18.

5. Hambleton, R. K. Fundamentals of item response theory / R. K. Hambleton, H. Swaminathan, H. J. Rogers. London : SAGE Publications, 1991. - 175 p.

6. Boone, W. J. Rasch Analysis in the Human Sciences / W. J. Boone, J. R. Staver, M. S. Yale. - New York, London : Springer, 2014. $-482 \mathrm{p}$.

7. Луговская, А. А. Анализ конструктной валидности и надежности шкал методики диагностики копинг-механизмов Э. Хайма на основе модели Раша / А. А. Луговская // Журнал Гродненского государственного медицинского университета. - 2017. - № 1 (57). - C. 42-47.

8. Baghaei, P. The Rasch Model as a Construct Validation Tool / P. Baghaei // Rasch Measurement Transactions. 2008. - Vol. 22, № 1. - P. 1145-1146.

9. Wright, B. D. Reliability and separation / B. D. Wright // Rasch Measurement Transactions. - 1996. - Vol. 9, № 4. - P. 472.

\section{References}

1. Fer RM, Bakarak VR. Psihometrika. Vvedenie. Cheljabinsk : Izdatel'skij centr JuUrGU; 2010. 445 p. (Russian).

2. Nabiullina RR, Tuhtarova IV. Mehanizmy psihologicheskoj zashhity i sovladanija so stressom (opredelenie, struktura, funkcii, vidy, psihoterapevticheskaja korrekcija). Kazan': Kazanskaja Gosudarstvennaja Medicinskaja Akademija; 2003. p. 17-25. (Russian).

3. Assanovich MA. Optimizacija shkaly ocenki depressii Gamil'tona na osnove modeli Rasha. Medicinskaja psihologija v Rossii. 2015;2(31):7. Available at: http://www. medpsy.ru/mprj/archiv_global/2015_2_31/nomer09.php (accessed 20.10.2016). (Russian).

4. Assanovich MA. Statisticheskoe obosnovanie kriteriev ocenki vyrazhennosti izmerjaemogo konstrukta v klinicheskoj psihodiagnostike (na primere testa nervno-psihicheskoj adaptacii) [Statistical grounding of severity criteria of measuring construct in clinical psychodiagnostics (for example, neuropsychic adaptation test)]. Psihiatrija, psihoterapija i klinicheskaja psihologija [Psychiatry, psychotherapy and clinical psychology]. 2014;2(16):9-18. (Russian).

5. Hambleton RK, Swaminathan H, Rogers HJ. Fundamentals of item response theory. London: SAGE Publications; 1991. $175 \mathrm{p}$.

6. Boone WJ, Staver JR, Yale MS. Rasch Analysis in the Human Sciences. New York, London: Springer; 2014. 482 p.

7. Lugovskaya AA. Analiz konstruktnoj validnosti i nadezhnosti shkal metodiki diagnostiki koping-mehanizmov Je. Hajma na osnove modeli Rasha [Psychometrical properties of the scale of constructive strategies of copying behavior diagnostics methods]. Zhurnal Grodnenskogo gosudarstvennogo medicinskogo universiteta [Journal of the Grodno State Medical University]. 2017;1(57):42-47. (Russian).

8. Baghaei P. The Rasch Model as a Construct Validation Tool. Rasch Measurement Transactions. 2008;22(1):11451146.

9. WrightBD. Reliability and separation. Rasch Measurement Transactions. 1996;9(4):472.

\title{
PSYCHOMETRIC PROPERTIES OF THE SCALE OF CONSTRUCTIVE STRATEGIES OF THE COPING BEHAVIOR DIAGNOSTIC TECHNIQUE
}

\author{
Lugorskaya A. A.
}

Educational Institution «Grodno State Medical University», Grodno, Belarus

Background. The problem of coping behavior diagnostics is of particular interest due to the requirements of modern psychometrics. At the moment, diagnostic methods of this construct with reasonable psychometric characteristics are lacking in clinical practice.

Purpose. The research purpose was to analyze the psychometric properties of the scale of constructive strategies of the coping behavior questionnaire based on the Rasch model.

Material and methods. A total of 270 respondents were assessed with the help of the coping behavior diagnostic technique. The difficulty of the scale items was calculated. The construct validity of the scale was assessed. The reliability and separation statistics values were determined.

Results. The scale item difficulty measures ranged from -1.04 to 1.20 logits. The UMS and WMS indices had acceptable values (in the range of $0.7-1.3$ ). The index of reliability was 0.81 .

Conclusions. The scale items of the designed questionnaire are balanced by the degree of difficulty. The construct validity of the items is sufficient. The index of reliability of the method has an acceptable significance (0.81). The using of the scale makes it possible to diagnose three levels of severity of the construct.

Keywords: coping mechanisms diagnostic technique, construct validity, reliability, Rasch model 